Supporting Information

\title{
Caribbean lead and mercury pollution archived in a crater lake
}

Colin A. Cooke ${ }^{1,2^{*}}$, Jason H. Curtis ${ }^{3}$, William F. Kenney ${ }^{4}$, Paul Drevnick ${ }^{5}$, and Peter E. Siegel ${ }^{6}$

${ }^{1}$ Department of Earth and Atmospheric Sciences, University of Alberta, Edmonton, AB, T6G

2E3, Canada; ORCID: 0000-0002-7417-5263

2 Environment and Parks, Government of Alberta, Edmonton, AB, T5J 5C6, Canada

${ }^{3}$ Department of Geological Sciences, University of Florida, Gainesville, FI 32611, United States

${ }^{4}$ Land Use and Environmental Change Institute, University of Florida, Gainesville, FI 32611, United States

${ }^{5}$ Environment and Parks, Government of Alberta, 3535 Research Road NW, Calgary, AB, T2L

2K8; ORCID: 0000-0003-2843-9720

${ }^{6}$ Department of Anthropology, Montclair State University, Montclair, NJ 07043, United States; ORCID: 0000-0003-1260-7646

* Corresponding author: cookeca@gmail.com

Additional methods and materials, 3 figures, 3 tables.

To adhere to the FAIR (Findability, Accessibility, Interoperability, and Reuse) data principles a raw data file is also included. 


\section{Additional methods and materials}

Normalization and the calculation of atmospheric deposition versus watershed inputs Land use and land cover changes occurred within the Lake Antoine watershed within in the industrial era. The watershed went from being covered by native vegetation to being covered by a patchwork of cultivated crops. This change in watershed cover increased the supply of terrigenous material to the lake. To subtract this erosional component from the overall records of $\mathrm{Pb}$ and $\mathrm{Hg}$ concentrations and accumulation rates, we used equations 1 and 2 below, respectively. ${ }^{1}$ The equations solve for the "excess" component of the sediment record, which in this case amounts to the atmospheric deposition amount.

(1) $\mathrm{Pb}_{\text {excess }}=\mathrm{Pb}_{x}-\mathrm{Al}_{x} \times(\mathrm{Pb} / \mathrm{Al})_{\text {preind }}$

(2) $\mathrm{Hg}_{\text {excess }}=\mathrm{Hg}_{x}-\mathrm{Hg}_{\text {preind, avg }} \times\left(\mathrm{OM}_{x} / \mathrm{OM}_{\text {preind, avg }}\right)$

Where $\mathrm{Pb}_{\text {excess }}$ and $\mathrm{Hg}_{\text {exces }}$ are the atmospheric deposition components, $\mathrm{Pb}_{x}, \mathrm{Al}_{x}, \mathrm{Hg}_{x}$ are the $\mathrm{Pb}_{\text {labile, }} \mathrm{Al}_{\text {total, }}$ and $\mathrm{Hg}$ concentrations in a given depth interval, $(\mathrm{Pb} / \mathrm{Al})_{\text {preind }}$ is the average ratio within preindustrial sediments (i.e., $1000 \mathrm{BC}$ to $1800 \mathrm{AD})$, and $\left(\mathrm{OM}_{x} / \mathrm{OM}_{\text {preind,avg }}\right)$ is the ratio of Organic Matter in a given depth interval relative to the mean Organic Matter content in preindustrial sediment. 


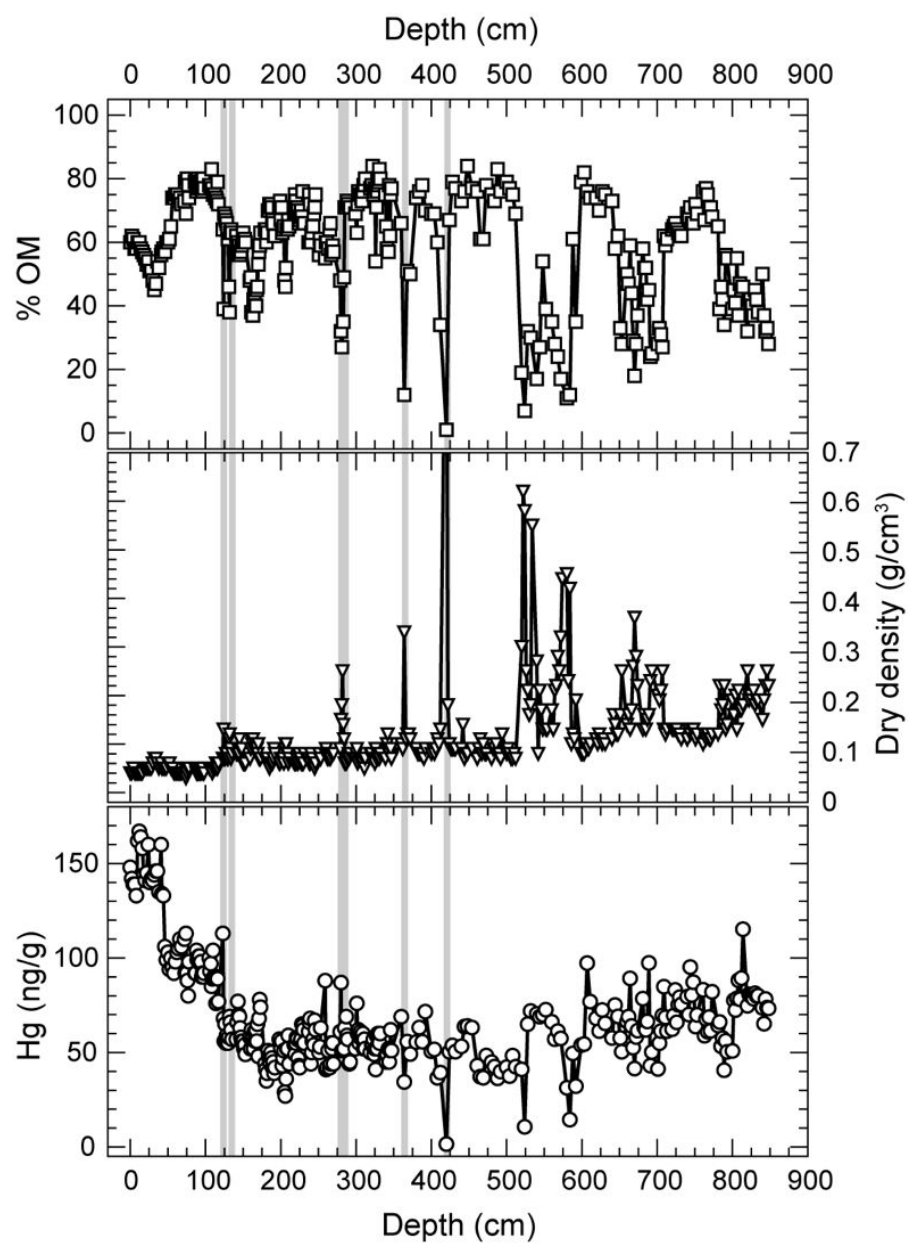

Figure S1. Profiles of \% organic matter (OM), dry bulk density, and Hg concentration in the Lake Antoine sediment core. Discrete horizons of tephra (grey shading) or the rapid in-wash of inorganic material register as abrupt decreases in \%OM and (most often) $\mathrm{Hg}$ and increases in dry bulk density. These events are thought to represent near instantaneous intervals of sedimentation and were therefore excluded from the final composite age-depth model and geochemical record. 


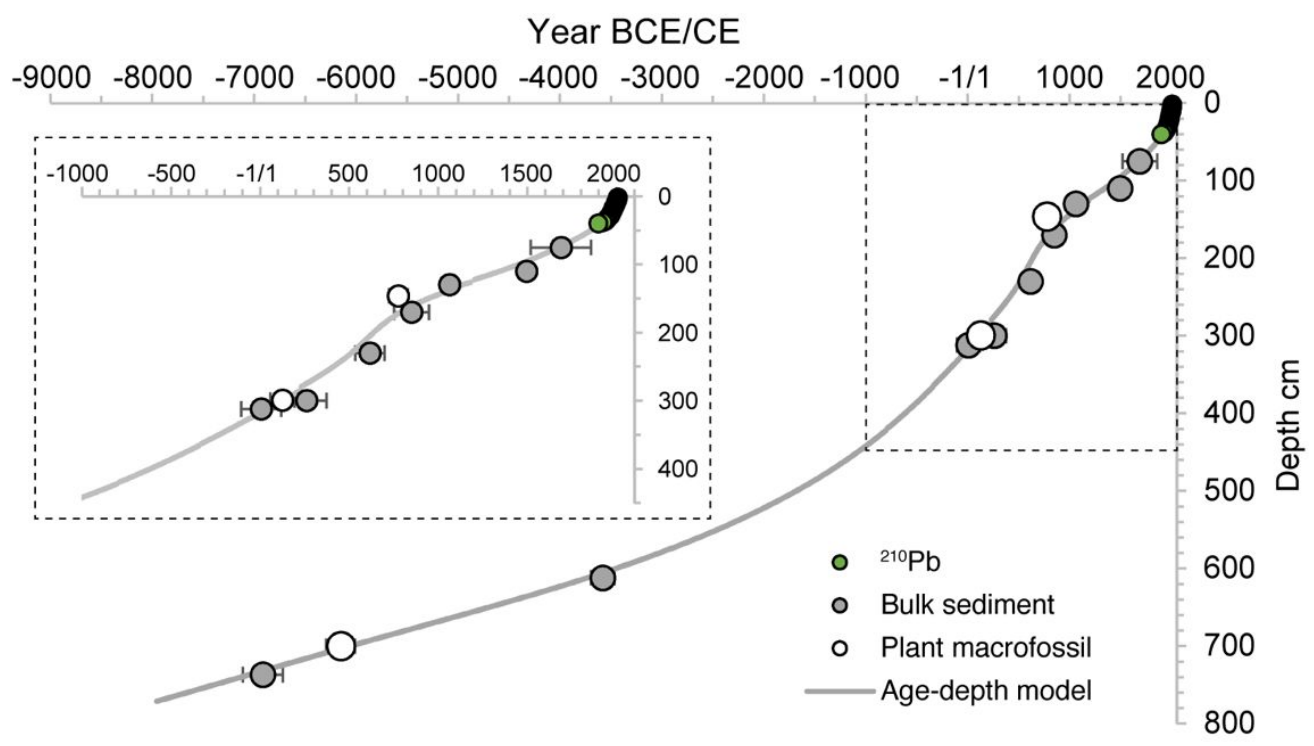

Figure S2. Age-depth model for the Lake Antoine sediment core integrating both ${ }^{210} \mathrm{~Pb}$ and ${ }^{14} \mathrm{C}$ ages. The age-depth model was generated using the CLAM package in $\mathrm{R}$ and intcal 20 calibration curve. CLAM calibrates the ${ }^{14} \mathrm{C}$ dates and then generates multiple age-depth curves through point estimates sampled from the (calibrated) dates (and the ${ }^{210} \mathrm{~Pb}$ dates). For each date, the probability of a calendar year being sampled is proportionate to its calibrated probability. Uncertainty ranges as well as a "best" age-depth model are calculated. The figure above shows the "best" age-depth model. The inset shows the past 3000 of lake history, which is the focus of this study. 


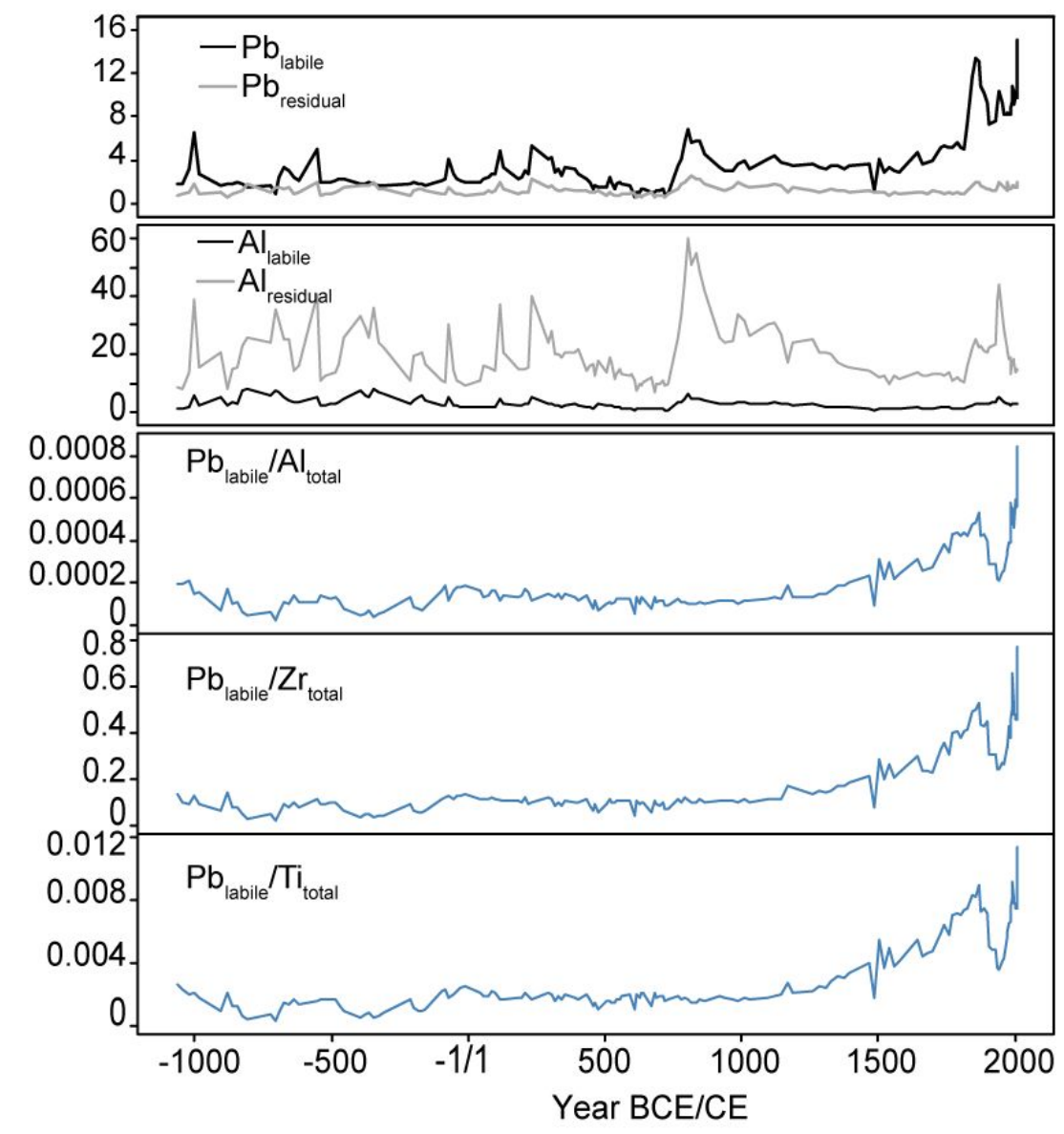

Figure S3. Plots of the labile and residual fractions of $\mathrm{Pb}$ and $\mathrm{Al}$ as well as $\mathrm{Pb}$ normalized to various lithogenic elements, including $\mathrm{Al}, \mathrm{Zr}$, and $\mathrm{Ti}$.

Table S1. ${ }^{210} \mathrm{~Pb}$ results for the Lake Antoine sediment core.

\begin{tabular}{ccccccc}
\hline $\begin{array}{c}\text { Depth } \\
\text { interval } \\
(\mathrm{cm})\end{array}$ & $\begin{array}{c}{ }^{210} \mathrm{~Pb} \\
\text { activity } \\
(\mathrm{dpm} / \mathrm{g})\end{array}$ & $\begin{array}{c}{ }^{210} \mathrm{~Pb} \text { error } \\
(1 \sigma)\end{array}$ & $\begin{array}{c}{ }^{226} \mathrm{Ra} \\
\text { activity } \\
(\mathrm{dpm} / \mathrm{g})\end{array}$ & $\begin{array}{c}{ }^{226} \mathrm{Ra} \text { error } \\
(1 \sigma)\end{array}$ & $\begin{array}{c}{ }^{137} \mathrm{Cs} \\
(\mathrm{dpm} / \mathrm{g})\end{array}$ & $\begin{array}{c}{ }^{137} \mathrm{Cs} \text { error } \\
(1 \sigma)\end{array}$ \\
\hline $0-2$ & 22.1 & 0.4 & 0.42 & 0.37 & 0.65 & 0.09 \\
$2-4$ & 18.0 & 0.3 & 0.27 & 0.24 & 0.87 & 0.10 \\
$4-6$ & 20.9 & 0.9 & 0.85 & 0.18 & 1.08 & 0.10 \\
$6-8$ & 21.4 & 0.5 & 0.51 & 0.48 & 1.45 & 0.14 \\
$8-10$ & 16.9 & 0.5 & 0.48 & 0.51 & 1.38 & 0.12 \\
$10-12$ & 19.8 & 0.3 & 0.31 & 0.42 & 1.43 & 0.12 \\
$12-14$ & 17.6 & 0.3 & 0.27 & 0.24 & 1.29 & 0.13 \\
$14-16$ & 17.1 & 0.1 & 0.15 & 0.18 & 1.73 & 0.12 \\
$16-18$ & 15.7 & 0.4 & 0.44 & 0.38 & 1.75 & 0.12 \\
$18-20$ & 12.1 & 0.2 & 0.20 & 0.18 & 2.31 & 0.10
\end{tabular}




\begin{tabular}{|c|c|c|c|c|c|c|}
\hline $20-22$ & 10.4 & 0.5 & 0.50 & 0.66 & 2.15 & 0.14 \\
\hline $22-24$ & 9.8 & 0.7 & 0.74 & 0.49 & 2.17 & 0.13 \\
\hline $24-26$ & 10.3 & 0.2 & 0.20 & 0.18 & 2.81 & 0.15 \\
\hline $26-28$ & 9.5 & 0.3 & 0.34 & 0.36 & 2.76 & 0.12 \\
\hline $28-30$ & 8.9 & 0.3 & 0.29 & 0.25 & 2.71 & 0.07 \\
\hline $30-32$ & 8.6 & 0.4 & 0.42 & 0.36 & 2.10 & 0.10 \\
\hline $32-34$ & 7.0 & 0.5 & 0.50 & 0.44 & 2.00 & 0.08 \\
\hline $34-36$ & 6.9 & 0.6 & 0.56 & 0.21 & 1.64 & 0.08 \\
\hline $36-38$ & 8.3 & 0.4 & 0.36 & 0.31 & 0.87 & 0.07 \\
\hline 39-41 & 9.0 & 0.6 & 0.59 & 0.04 & 1.48 & 0.10 \\
\hline $40-42$ & 3.1 & 0.2 & 0.18 & 0.31 & 0.48 & 0.05 \\
\hline $42-44$ & 2.9 & 0.5 & 0.47 & 0.09 & 0.17 & 0.03 \\
\hline $44-46$ & 1.7 & 0.4 & 0.37 & 0.23 & 0.32 & 0.03 \\
\hline $46-48$ & 1.9 & 0.4 & 0.39 & 0.18 & 0.34 & 0.04 \\
\hline $48-50$ & 3.1 & 0.5 & 0.48 & 0.07 & 0.46 & 0.05 \\
\hline $50-52$ & 1.8 & 0.6 & 0.62 & 0.06 & 0.40 & 0.04 \\
\hline $52-54$ & 1.9 & 0.3 & 0.28 & 0.08 & 0.17 & 0.03 \\
\hline $54-56$ & 1.0 & 0.2 & 0.21 & 0.06 & 0.12 & 0.02 \\
\hline $56-58$ & 0.7 & 0.3 & 0.34 & 0.19 & 0.19 & 0.03 \\
\hline $58-60$ & 1.9 & 0.3 & 0.34 & 0.36 & 0.25 & 0.04 \\
\hline $60-62$ & 1.4 & 0.2 & 0.20 & 0.13 & 0.02 & 0.01 \\
\hline $62-64$ & 1.3 & 0.2 & 0.17 & 0.15 & 0.30 & 0.03 \\
\hline 64-66 & 2.3 & 0.1 & 0.13 & 0.14 & 0.31 & 0.03 \\
\hline $66-68$ & 1.3 & 0.4 & 0.41 & 0.19 & 0.42 & 0.03 \\
\hline $68-70$ & 2.1 & 0.4 & 0.39 & 0.17 & 0.24 & 0.03 \\
\hline 70-74 & 1.3 & 0.0 & 0.04 & 0.04 & 0.23 & 0.03 \\
\hline $80-84$ & 1.0 & 0.3 & 0.28 & 0.11 & 0.03 & 0.01 \\
\hline 88-92 & 1.7 & 0.2 & 0.19 & 0.12 & 0.25 & 0.02 \\
\hline $92-94$ & 1.0 & 0.2 & 0.18 & 0.17 & 0.30 & 0.04 \\
\hline 108-110 & 0.4 & 0.4 & 0.39 & 0.13 & 0.04 & 0.01 \\
\hline $122-124$ & 0.7 & 0.5 & 0.54 & 0.13 & 0.10 & 0.02 \\
\hline
\end{tabular}


Table S2. Radiocarbon $\left({ }^{14} \mathrm{C}\right)$ dates in years before present (BP) used as part of this study.

\begin{tabular}{llllll}
\hline Lab Sample \# & Drive & $\begin{array}{l}\text { Drive mid- } \\
\text { depth }(\mathrm{cm})\end{array}$ & $\begin{array}{l}\text { Composite } \\
\text { depth }(\mathrm{cm})\end{array}$ & $\begin{array}{l}{ }^{14} \mathrm{C} \text { Age BP } \\
(1 \sigma)\end{array}$ & $\begin{array}{l}\text { Material } \\
\text { dated }\end{array}$ \\
\hline D-AMS 029992 & 1 & 25 & 75 & $295( \pm 25)$ & sediment \\
D-AMS 029993 & 1 & 60 & 110 & $460( \pm 30)$ & sediment \\
D-AMS 029994 & 1 & 80 & 130 & $1025( \pm 25)$ & sediment \\
Beta-377885 & 1 & 96 & 146 & $1290( \pm 30)$ & plant \\
D-AMS 029995 & 2 & 20 & 170 & $1215( \pm 30)$ & sediment \\
D-AMS 029996 & 2 & 80 & 230 & $1495( \pm 25)$ & sediment \\
D-AMS 029998 & 3 & 49 & 299 & $1920( \pm 30)$ & wood \\
D-AMS 029997 & 3 & 50 & 300 & $1825( \pm 30)$ & sediment \\
AA91729 & 3 & 62 & 312 & $2030( \pm 40)$ & sediment \\
AA91728 & 6 & 62 & 612 & $4860( \pm 45)$ & sediment \\
Beta-377883 & 7 & 50 & 700 & $7340( \pm 40)$ & plant \\
AA91730 & 7 & 87 & 737 & $8055( \pm 50)$ & sediment \\
\hline
\end{tabular}


Table S3. Results of QC samples included as part of the $\mathrm{Hg}$ and trace element measurements. Certified concentrations for the reference materials (MESS-3 and MESS-4: Marine Sediment; GSP-2: Granodiorite) are included in the table with their $1 \sigma$ uncertainty ranges (where available) and apply to the total concentration (labile plus residual fractions). Duplicate results represent distinct samples digested and analyzed separately and are expressed as the relative percent difference (RPD) from one another. Insufficient sediment remained for duplicate $\mathrm{Hg}$ measurements; however, the long-term (>2 year) University of Alberta laboratory average is < $5 \%$ for lake sediment matrices.

\begin{tabular}{lllll}
\hline $\begin{array}{l}\text { Element } \\
\text { concentration } \\
(\mathrm{mg} / \mathrm{kg})\end{array}$ & $\begin{array}{l}\text { Certified } \\
\text { concentration } \\
(\mathrm{mg} / \mathrm{kg})\end{array}$ & $\begin{array}{l}\text { Measured } \\
\text { concentration } \\
(\mathrm{mg} / \mathrm{kg})\end{array}$ & $\begin{array}{l}\% \\
\text { recovery }\end{array}$ & $\mathrm{n}$ \\
\hline $\mathrm{Hg} \mathrm{MESS}-3$ & $91( \pm 9)$ & $91( \pm 1)$ & 100 & 6 \\
$\mathrm{Hg}$ MESS-4 & $80( \pm 60)$ & $53( \pm 1)$ & 100 & 38 \\
Total Al MESS-4 & $79100( \pm 2000)$ & $58015( \pm 10989)$ & 73 & 14 \\
Total Al GSP-2 & 78800 & $49349( \pm 13140)$ & 63 & 5 \\
Total Pb MESS-4 & $21.5( \pm 1.2)$ & $22.8( \pm 0.5)$ & 106 & 14 \\
Total Pb GSP-2 & 42 & $39( \pm 2)$ & 94 & 5 \\
Total Ti MESS-4 & $3840( \pm 220)$ & $3420( \pm 193)$ & 89 & 14 \\
Total Ti GSP-2 & 4000 & $3568( \pm 183)$ & 89 & 5 \\
\hline
\end{tabular}

\begin{tabular}{lll}
\hline Element & Average RPD & $\mathrm{n}$ \\
\hline $\mathrm{Hg}$ & $\mathrm{n} / \mathrm{a}$ & 0 \\
Labile $\mathrm{Al}$ & 2.2 & 13 \\
Labile $\mathrm{Pb}$ & 1.3 & 13 \\
Labile $\mathrm{Ti}$ & 0.7 & 13 \\
Labile $\mathrm{Zr}$ & 18.9 & 13 \\
Residual $\mathrm{Al}$ & 1.5 & 15 \\
Residual $\mathrm{Pb}$ & 2.6 & 15 \\
Residual $\mathrm{Ti}$ & 1.1 & 15 \\
Residual $\mathrm{Zr}$ & 2.4 & 15 \\
\hline
\end{tabular}

